\title{
Improved Therapies for HIV Infection and AIDS-Implications for Primary Care
}

In this issue of The Fournal, Goldschmidt and colleagues ${ }^{1}$ once again nicely summarize the many treatment choices available to care for persons infected with human immunodeficiency virus (HIV). In their introduction the authors note that HIV infection is becoming a chronic disease; the implication is that clinicians must approach management of HIV infection differently than we did a decade ago. To be sure, in the 1980 s, we had limited management options. Mostly, we applied our energies to prevent HIV transmission and to prevent the complications of HIV infection and acquired immunodeficiency syndrome (AIDS). Persons at risk for HIV infection were strongly encouraged to reduce their risks (safer sex, not sharing needles). To persons already infected, we provided immunizations, prophylaxis and treatment for opportunistic infections, and behavioral prevention strategies (quit smoking, use safer sex to protect partners and avoid new infections). When antiretroviral therapy became available, our early experience reaffirmed the need to prioritize primary prevention. New therapies available in the 1990 s, however, are proving remarkably effective for many HIV-infected persons. Patients appear to be living longer and better lives. ${ }^{2}$ So is HIV infection becoming just another chronic disease?

We believe HIV infection is becoming a paradigm for modern disease, transcending the notions of acute versus chronic or even of infectious versus noninfectious disease. Increasingly, all diseases of modern life appear to have important behavioral, genetic, and environmental (including infectious) causes. Behavioral risks are evident for virtually all

Submitted 1 September 1998.

From the HIV/AIDS Program, Seattle-King County Department of Public Health, Seattle (GMG, RWV), and the Departments of Epidemiology (GMG) and Medicine (RWW), University of Washington, Seattle. Reprint requests to Gary Goldbaum, MD, MPH, HIV/AIDS Program, Seattle-King County Department of Public Health, 1001 Broadway, Suite 218, Seattle, WA 98122. diseases. The new tools of genetic research are rapidly revealing genetic factors predisposing a person to cancer or heart disease. Increasingly, the evidence mounts that infection plays a role in many chronic diseases. Human papillomavirus has been associated with cervical cancer. Chlamydia might contribute to cardiovascular disease. There is speculation that diabetes and multiple sclerosis might have infectious causes.

HIV acquisition and progression reflect all of these components. Treating HIV infection also epitomizes the management of modern disease. Management is complex and expensive, demands integrated services (clinical and social), and ultimately hinges on behavior - success is achieved only if the patient adheres carefully to treatment regimens. ${ }^{3}$ Although this approach seems little different from the management of most cancers or cardiovascular disease; HIV is different in at least two crucial respects: (1) the infected person can infect others through activities (sex and sharing of injection equipment) that generally imply another's consent, and (2) HIV infection remains potentially more lethal than most other infectious diseases. These distinctions carry important implications for clinicians, patients, and society.

Primary prevention remains the mainstay of our efforts to control HIV infection. Just as we believe it is better to help people to not smoke than to treat heart disease or lung cancer, we must believe it is better to help people avoid HIV infection. For all the improvements, treatment of HIV infection is very difficult. Patients must take many pills, often in complicated schedules that completely interrupt their lives. Side effects are common and can be challenging ( $\mathrm{gg}$, buffalo hump); the long-term consequences of current therapies are largely unknown. Viral resistance is a major concern. Furthermore, the constant monitoring can prove emotionally challenging to clinician and patient alike ("What do you mean my viral load has gone up?"). 
In short, the new therapies offer hope but are not a reason to redirect our efforts. Although the HIV epidemic among men who have sex with men appears to have become endemic, the epidemic continues to expand among injection-drug users in developed countries and among heterosexuals worldwide. ${ }^{4}$ Indeed, it seems that preventing HIV infection is more important than ever. Moreover, clinicians have not done as well as we might to assess risk and counsel about reducing risk. ${ }^{5}$ Some physicians might not feel comfortable managing HIV infection, but we should all be comfortable with and aggressively offer risk-reduction counseling. To do so is not easy, especially as our patients have become numb to risk-reduction messages. Nonetheless, repeated reminders by clinicians can affect behaviors of some patients and are therefore well worth trying.

A corollary to the primary prevention theme is that we must help our HIV-infected patients avoid placing others at risk. This challenge is not simply altruistic. HIV-infected persons can become coinfected with other organisms (such as herpesvirus) that progress more rapidly than in those not infected with HIV. Coinfection with resistant strains of HIV has been documented and could represent a serious threat to effective management of HIV infection. ${ }^{6,7}$ Moreover, we are all physicians to a community broader than simply our own practices. The partners of our patients deserve protection. Although we might believe that these partners are consenting to placing themselves at risk for fatal diseases, the complex and secretive nature of sex and drug use means that consent rarely is explicit and might not be implied. Particularly in high-risk public sex environments (such as gay bath houses), negotiations can be limited to gestures. In a world where sex is promoted everywhere, public risk-reduction messages are rare. Clinicians can play a major role in helping their HIV-infected patients disclose their HIV status when negotiating sex or sharing injection equipment. Disclosure is not easy-negotiating to use condoms is hard enough, but disclosing HIV status can be terrifying ("Will this potential lover turn on me?"). Nonetheless, family physicians are uniquely positioned to influence the choices of HIV-infected persons.

An important adjunct to primary HIV prevention strategies is aggressive pursuit of other sexually transmitted diseases (STDs). HIV is more easily transmitted in the presence of other STDs, particularly ulcerating STDs; other STDs are also more easily transmitted and can progress more rapidly in the presence of HIV infection. ${ }^{8}$ It is not obvious that the new treatments will mitigate this potential; therefore, attending to HIV-infection management requires that we continue to attend to STD prevention generally.

A slightly different theme is that the new treatments are raising unexpected challenges to the notion of primary prevention. For example, there is evidence that prophylaxis with antiretroviral drugs can reduce the likelihood of infection among health care workers exposed parenterally to HIV. ${ }^{9}$ It is not clear that prophylaxis will prevent sexual transmission, but it is a reasonable conjecture. ${ }^{10}$ Should we invest heavily in postexposure prophylaxis? It is costly (perhaps $\$ 1000$ for 1 month), but it might be cheaper than caring for infected persons. ${ }^{11}$ Even so, who should pay? Perhaps more difficult is the potential for postexposure prophylaxis to lead persons to take more risks. Indeed, this possibility is a concern with respect to the general perception that HIV infection can be so effectively managed that it is no longer a fatal disease, merely an inconvenient one.

Family physicians need to understand how to care for HIV-infected persons. Even physicians who never manage HIV infections should appreciate the principles of management of modern disease, for which HIV infection is a model. More to the point, the improved therapies for HIV infection challenge us to reconsider the role of primary prevention and our responsibilities to our patients, their partners, and our community generally. Times have changed, but the family physician can and should take the lead in managing HIV disease at the community level, which means primary prevention. As we counsel our patients about the new therapies, we must also counsel about preventing transmission. For the moment there is still no cure-only prevention if we want to see our patients living long and healthy lives.

Gary M. Goldbaum, MD, MPH Robert W. Wood, MD University of Washington Seattle

\section{References}

1. Goldschmidt RH, Dong BJ. Treatment of AIDS and HIV-related conditions-1999. J Am Board Fam Pract 1999; 12:71-94. 
2. Palella FJ Jr, Delaney KM, Moorman AC, Loveless MO, Fuhrer J, Satten GA, et al. Declining morbidity and mortality among patients with advanced immunodeficiency virus infection. N Engl J Med 1998; 338:853-60.

3. Burman WJ, Reves RR, Cohn DL. The case for conservative management of early HIV disease. JAMA 1998; 280:93-5.

4. UNAIDS. The Joint United Nations Programme on HIV/AIDS. Report on the global HIV/AIDS epidemic: June 1998. (www.us.unaids.org)

5. Makadon HJ, Silin JG. Prevention of HIV infection in primary care: current practices, future possibilities. Ann Intern Med 1995;123:715-9.

6. Hecht FM, Grant RM, Petropoulos CJ, Dillon B, Chesney MA, Tian H, et al. Sexual transmission of an $H I V-1$ variant resistant to multiple reverse-transcriptase and protease inhibitors. $\mathrm{N}$ Engl J Med 1998;339:307-11.

7. Cohen OJ, Fauci AS. Transmission of multidrug-resistant human immunodeficiency virus, the wake-up call. N Engl J Med 1998;339:341-3.

8. HIV prevention through early detection and treatment of other sexually transmitted diseases-United States. Recommendations of the Advisory Committee for HIV and STD prevention. MMWR Morb Mortal Weekly Rep 1998;47(RR-12):1-24.

9. Case-control study of HIV seroconversion in healthcare workers after percutaneous exposure to HIV-infected blood-France, United Kingdom, and United States, January 1988-August 1994. MMWR Morb Mortal Weekly Rep 1995;44:929-33.

10. Katz MH, Gerberding JL. Postexposure treatment of people exposed to the human immunodeficiency virus through sexual contact or injection-drug use. $N$ Engl J Med 1997;336:1097-100.

11. Pinkerton SD, Holtgrave DR, Pinkerton HJ. Costeffectiveness of chemoprophylaxis after occupational exposure to HIV. Arch Intern Med 1997;157: 1972-80.

\section{The Tyranny of Names in Mental Health Care}

Yogi Berra's wisdom often extends beyond baseball. He once said that 90 percent of baseball is half mental, but he could have as easily said that 90 percent of family practice is half mental illness. Family physicians spend a considerable portion of their time and effort in the diagnosis and treatment of emotional distress and clinical psychiatric

Submitted 10 November 1998.

From the Department of Family Medicine, University of Michigan Health System, Ann Arbor, Michigan. Address reprint requests to Thomas $L$. Schwenk, MD, Department of Family Medicine, 1018 Fuller St, Ann Arbor, MI 48109. disease. ${ }^{1}$ Most prescriptions for psychotropic medications are written by family physicians, ${ }^{2}$ and most patients who receive mental illness care do so from primary care physicians rather than specialty mental health services, leading to the labeling of primary care as the "hidden mental health care system."3

The occurrence of both distress and mood and anxiety disorders in primary care is high, their functional impact profound, their presence frequently undiagnosed by primary care and family physicians, and their diagnosis often deliberately miscoded because of insurance restrictions and social stigma. ${ }^{4}$ These problems and deficiencies have led to a rigorous, but increasingly unproductive, line of linear reasoning that suggests the major solution to this epidemic of undiagnosed and presumably untreated mental illness is to exhort physicians to perform better through education about diagnostic criteria and appropriate treatment, usually in the form of guidelines and protocols. 5

Recent studies, however, have suggested the futility of these educational exhortations, as well as the reasons for this futility. Many cases of depression, for example, might meet diagnostic criteria but are mild and cause little functional impact. ${ }^{6}$ Family physicians have competing priorities for the care of most patients and must decide which of several problems deserve attention during a short visit. ${ }^{7}$ Depression often is watched or otherwise not treated in a specific fashion because of patient resistance and the presence of seemingly more serious problems. Treatment provided to many depressed primary care patients does not lead to improvement, and patients whose depression is not diagnosed often do as well as or better than those whose depression is diagnosed, because of the waxing and waning course of depression in primary care. ${ }^{8}$ Prognosis has more to do with whether the patient has a chronic medical disease and the patient's marital and social support than the specific nature of the depression treatment. ${ }^{9}$ In sum, depression as it is known, described, labeled, and depicted in psychiatry might have little in common with the disease that carries the same name in primary care.

We are prisoners of how medical problems are named. The clinical skills and outcomes of primary care physicians have been unfairly compared with those of psychiatrists and mental health workers 